\title{
Neuropsychological effects of antiepileptic drugs (carbamazepine versus valproate) in adult males with epilepsy
}

This article was published in the following Dove Press journal:

Neuropsychiatric Disease and Treatment

14 October 2009

Number of times this article has been viewed

\author{
Ghaydaa A Shehata' \\ Abd El-aziz M Bateh ${ }^{2}$ \\ Sherifa A Hamed' \\ Tarek A Rageh' \\ Yaser B Elsorogy' \\ 'Department of Neurology \\ and Psychiatry, Faculty of Medicine, \\ Assiut University, Egypt; ${ }^{2}$ Department \\ of Psychology, Faculty of Arts, Banha \\ University, Egypt
}

Purpose: To evaluate the effect of antiepileptic drugs (AEDs) on cognition and behavior in adult epileptic males controlled on treatment with conventional antiepileptic medications.

Methods: Cognitive, mood, behavior and personality traits were assessed in 45 epileptic patients treated with carbamazepine and/or valproate and free of seizures for $\geq 1$ year. Thirtyfour newly diagnosed or untreated patients with epilepsy and 58 matched healthy subjects were also included for comparison. A battery of psychometric tests was utilized including StanfordBinet (4th edition), Beck Inventory for Depression, Aggressive Scale and Eysenck Personality Questionnaire.

Results: Compared to matched control subjects, treated and untreated epileptic patients had poor performance in different cognitive and behavioral functions testing. Treated patients had worse scores in memory for digits forward and backward, total short-term memory, extroversion and psychosis. The duration of AEDs intake was correlated with memory of objects $(\mathrm{r}=-0.323 ; P=0.030)$, bead memory $(\mathrm{r}=-0.314 ; P=0.036)$ and total nonverbal short-term memory $(\mathrm{r}=-0.346 ; P=0.020)$. Treated and untreated epileptic patients had poor performance of similar extent in behavioral functions testing (depression, aggression and neurosis). The dose of AEDs was correlated with testing scores for neurosis $(\mathrm{r}=0.307 ; P=0.040)$, verbal aggression $(\mathrm{r}=0.483 ; P=0.001)$ and nonverbal aggression $(\mathrm{r}=0.526 ; P=0.000)$, and duration of drug intake was correlated with scores for depression $(\mathrm{r}=0.384 ; P=0.009)$, psychosis $(\mathrm{r}=0.586$; $P=0.0001)$ and nonverbal aggression $(\mathrm{r}=0.300 ; P=0.045)$.

Conclusions: This study provides support for the notion that AEDs can impair performance in cognition, mood and behavior. Duration of drug intake and the number of the utilized AEDs are the main confounding variables. This study did not provide clues on how to exclude the effect of epilepsy itself and psychosocial variables as additional important confounding variables.

Keywords: cognition, behavior, mood, personality traits, epilepsy, AEDs

\section{Introduction}

Epilepsy is a common health problem. ${ }^{1}$ Seizures are only one aspect of epilepsy. Patients with epilepsy may experience cognitive and behavioral problems that may have a deleterious impact on patients' overall lives. ${ }^{2,3}$ Cognitive comorbidity associated with epilepsy has been confirmed in clinical, experimental, pathological, psychological, physiological and imaging studies. ${ }^{4-6}$ Epileptic patients may experience problems in various cognitive domains such as reduced intelligence, attention, problems in memory, language and frontal executive functions, despite the side or site of lesion. The association between epilepsy and poor cognitive performance, learning and longterm memory is correlated with a number of variables including genetics, basic brain lesion, site and side of structural brain lesion, and age at onset together with duration of
Correspondence: Ghaydaa Ahmed Shehata Lecturer in Neurology, Department of Neurology and Psychiatry, Assiut University Hospital, PO Box

71516, Assiut, Egypt

$\mathrm{Tel}+20882297075$

Fax +2 0882333327

Email ghaydaa83@yahoo.com 
epilepsy, seizure frequency, adverse effects from antiepileptic medications and psychosocial variables..$^{7-10}$

Antiepileptic drugs (AEDs) have both negative and positive effects on cognition and behavior. ${ }^{5}$ AEDs are able to improve cognition and behavior, which has been attributed to reduction of seizure activity, and modulating effect on neurotransmitters and their psychotrophic effect. AEDs reduce neuronal irritability and increase postsynaptic inhibition or alter synchronization of neural networks to decrease excessive neuronal excitability associated with seizure development and secondary spread of epileptic activity to the surrounding normal brain. However, excessive reduction of neuronal excitability may result in slowed motor and psychomotor speeds, and poor attention and memory processing, which are common side effects of sodium channel blockade and increasing GABAergic inhibitory activity. ${ }^{11-13}$ It is not surprising that patients with epilepsy are more susceptible to the adverse behavioral effects of AEDs than other populations, possibly due to the disease associated structural or functional changes that increase their risk of psychiatric disorders.

\section{Aim of the work}

This study aimed to evaluate the effect of conventional AEDs (carbamazepine [CBZ] and/or valproate [VPA]) on cognitive and behavioral functions in a group of adult epileptic males with generalized tonic-clonic convulsions and controlled (seizure free for $\geq 1$ year) on AEDs. Correlations between cognitive and behavioral functions and variables related to AEDs (dose, duration of treatment and number of utilized AEDs) were also determined.

\section{Patients and methods}

This study included 79 consecutive patients (range 18 to 45) with generalized tonic-clonic convulsions (grand mal epilepsy) recruited from the outpatient epilepsy clinic of Assuit University Hospital, Assuit, Egypt. Epilepsy type was defined according to the International League Against Epilepsy (1989). ${ }^{14}$ The protocol of the study was in conformity with ethical guidelines of Assiut University Hospital, Egypt and informed written consent was obtained from each participant. Included in this study were: a) newly diagnosed and untreated epileptic patients: these were patients with at least one seizure during the last 6 months and who had not received AEDs $(n=34)$; b) treated epileptic patients: these were patients on regular treatment with one (monotherapy) or combined (polytherapy) AEDs (total: $n=45 ; C B Z: n=25 ;$ VPA; $n=13 ; C B Z+V P A: n=7$ ).
AEDs were described according to the well-known guidelines and in recommended doses. All treated patients gave a history of compliance to AEDs. Compliance was confirmed by assessment of the serum drug level at least once during the period of the study. The serum levels of AEDs were determined in the therapeutic drug monitoring lab, Assiut University Hospital, Assiut Egypt, with the fluorescence polarization immunoassay system of Abbott (EPIA) using TD $\times$ FLX apparatus (Abbott Lab, Wiesbaden, Germany), as described before. ${ }^{15}$ This study included 58 healthy males matched for age, sex, educational level and socioeconomic status as controls (Table1). Excluded from this study were a) patients with intelligence quotient (IQ) less than 70 assessed using Wechsler Adults Intelligence ScaleRevised; ${ }^{16}$ b) presence of history of head injury; c) patients with progressive central nervous system (CNS), psychiatric or systemic medical conditions that might affect cognitive or behavioral functions; and d) patients utilizing chronic medications other than AEDs. The demographic and clinical characteristics of the studied groups are summarized in Table 1.

All participants were subjected to:

1. Complete neurological, medical history and examination, and socioeconomic status scale assessment. ${ }^{17}$

2. Analysis of seizure history included: age at onset, duration of epilepsy, type and duration of AED(s) utilized and the degree patients' control on AED(s). Only controlled patients on AEDs (seizure free for $\geq 1$ year) were included in this study.

3. Neuropsychological assessment

(a) Assessment of cognition

Cognition was assessed using the standardized and validated Arabic version of the Stanford-Binet Test (4th edition). ${ }^{18,19}$ The test consisted of 30 items ranging from the ability to touch one's nose or ear when asked, to the ability to draw designs from memory and to define abstract concepts. The following items were tested and analyzed: total verbal reasoning, which was used to assess vocabulary, comprehension, absurdities and verbal relations; total nonverbal short-term memory, which tested memory for objects and bead memory; and total verbal short-term memory, which was used to assess memory for digits (forward and backward) and memory for sentences.

(b) Behavioral assessment

A standardized and validated Arabic versions of the Beck Depression Inventory, ${ }^{20,21}$ the Aggressive Behavior Scale (Verbal and Nonverbal) ${ }^{22,23}$ and Eysenck Personality Inventory ${ }^{24,25}$ were used for behavioral and emotional 
Table I Demographic and clinical characteristics of the studied groups

\begin{tabular}{|c|c|c|c|}
\hline & $\begin{array}{l}\text { Controls } \\
(n=58)\end{array}$ & $\begin{array}{l}\text { Untreated } \\
(n=34)\end{array}$ & $\begin{array}{l}\text { Treated } \\
(n=45)\end{array}$ \\
\hline Age, years & $30.45 \pm 7.56$ & $28.09 \pm 8.99$ & $28.80 \pm 7.69$ \\
\hline Socioeconomic status & $10.20 \pm 3.914$ & $9.68 \pm 3.96$ & $9.53 \pm 3.75$ \\
\hline Number of years of education & $9.03 \pm 5.67$ & $7.91 \pm 4.33$ & $9.04 \pm 3.74$ \\
\hline Intelligent quotient & $85.72 \pm 15.79$ & $78.24 \pm 20.13$ & $88.13 \pm 20.46$ \\
\hline$P$ value & & & 0.035 \\
\hline Duration of illness; years & - & $11.35 \pm 7.65$ & $10.07 \pm 6.59$ \\
\hline Family history of epilepsy & - & $5(14.7 \%)$ & II (24.4\%) \\
\hline History of febrile convulsions & & $2(5.9 \%)$ & $3(6.7 \%)$ \\
\hline Family history of cognitive decline & - & I (3\%) & $3(6.8 \%)$ \\
\hline Duration of drug intake, years & - & - & $8.54 \pm 6.52$ \\
\hline AEDs dose, $\mathrm{mg} /$ day & - & - & \\
\hline CBZ & & & $739.39 \pm 289.33$ \\
\hline VPA & & & $822.22 \pm 298.14$ \\
\hline
\end{tabular}

Notes: Data are expressed as mean \pm SD, number (\%).

Significance: between untreated and treated groups.

Abbreviations: AED, antiepileptic drug; CBZ, carbamazepine; VPA, valproate.

assessment. The Beck Depression Inventory is a 21-question multiple-choice self-report inventory, one of the most widely used instruments for measuring the severity of depression. Its development marked a shift among health care professionals, who had until then viewed depression from a psychodynamic perspective, instead of it being rooted in the patient's own thoughts. In its current version the questionnaire is designed for individuals aged 13 and over, and is composed of items related to symptoms of depression such as hopelessness and irritability, cognitions such as guilt or feelings of being punished, as well as physical symptoms such as fatigue, weight loss, and lack of interest in sex. The Aggressive Behavior Scale is a 64-item scale with excellent reliability and validity. It is used to assess verbal ( 32 items) and nonverbal aggressions (32 items). It measures levels of self-esteem, disinhibited behavior, aggression against persons and animals, control of rage, and so on. The scoring for each item ranges from 1 to 4 . Higher scores indicate higher levels of aggression. Eysenck Personality Inventory assesses neurosis, psychosis, extroversion-introversion, and lying. Neurosis is marked by excessive anxiety or apprehension that is not restricted to specific situations or objects, unlike anxiety experienced in threatening situations. Psychosis is a temporary mental state. A person experiences a psychotic state for a while, and then come out of it. Psychoticism focuses on the disturbances of such magnitude that there is personality disintegration and loss of contact with reality. Extraversion is marked by direction of attention and energy outward from the self. Lying is typically used to refer to deceptions in oral or written communication.

\section{Procedure}

Cognitive and behavioral assessment of the patients was done individually. Patients were divided into two smaller groups. The first subgroup completed the vocabulary, comprehension, absurdities, verbal relations, memory for objects, bead memory, memory for sentences, memory for digits forward, and memory for digits backward tests in that order. The second subgroup completed the same tests in reverse order. The aim of this counterbalance was to control the training variable in cognitive function. Behavioral assessment was completed in another session.

\section{Statistical analysis}

Descriptive statistics (mean, SD, and percentages) were calculated using the computer software package SPSS for Windows, Version 16. Results were analyzed using independent-sample $t$ test that did not assume equal variances. One-way analysis of variance (ANOVA) was followed by a post hoc test (LSD). Correlation coefficients and multiple linear regression analysis were used to detect the impact of epilepsy and its treatment on cognition, mood, aggressive behavior, and personality traits. The significance level was set at a $\leq 0.05$.

\section{Results}

Table 1 demonstrates the demographic and clinical characteristics of the studied groups. Tables 2 and 3 demonstrate the effect of AEDs on various cognitive and behavioral functions. Compared to matched control subjects, treated and untreated epileptic patients had poor performance in different cognitive and behavioral functions testing. However, treated patients 
had worse scores in memory for digits forward, memory for digits forward and backward, total short-term memory, extroversion and psychosis. Patients on VPA had worse scores in total aggression and verbal aggression scores compared to those on CBZ or combined treatment with CBZ.

In the treated group of patients, the dose of AEDs was significantly associated with testing scores for neurosis ( $\mathrm{r}=0.307 ; P=0.040)$, verbal aggression $(\mathrm{r}=0.483 ; P=0.001)$, nonverbal aggression $(\mathrm{r}=0.526 ; P=0.000)$. The duration of AED intake was significantly associated with testing scores for memory of objects $(\mathrm{r}=-0.323 ; P=0.030)$, bead memory $(\mathrm{r}=-0.314 ; P=0.036)$, total nonverbal short-term memory $(\mathrm{r}=-0.346 ; P=0.020)$, Beck Depression Inventory ( $\mathrm{r}=0.384 ; P=0.009)$, psychosis $(\mathrm{r}=0.586 ; P=0.0001)$ and nonverbal aggression $(\mathrm{r}=0.300 ; P=0.045)$. The number of AEDs was significantly correlated with testing scores for absurdities ( $\mathrm{r}=0.336 ; P=0.024)$ but not associated with other cognitive or behavioral functions.

\section{Discussion}

The potential for AEDs to adversely impact cognition and behavior is of significant concern as they are the major therapeutic modality for control of seizures. Even their modest effects can be of clinical significant and affect the patient's quality of life. ${ }^{26}$ In this study, we compared the results of cognitive and behavioral functions testing of a group of adult male patients who were seizure free for $\geq 1$ year on conventional AEDs (CBZ and VPA), with a group of epileptic patients who were newly diagnosed or did not ever received AEDs and a group of matched healthy subjects.

Others and ourselves have reported that epilepsy adverse affects different cognitive and behavioral functions. ${ }^{3,6,9,10,27}$ Not only ictal but also interictal (subclinical) discharges, which occur with paroxysmal focal or generalized epileptic activities, can disrupt cognitive encoding processes. ${ }^{28-30}$ In addition, neuronal plasticity, reorganization, sprouting and impairment of cellular metabolism associated with the process of epileptogenesis are fundamental determinants for progressive cognitive deterioration in epilepsy. ${ }^{31,32}$

The results of this study indicate that patients on conventional AEDs are at increased risk for cognitive dysfunction. In support, both treated and untreated epileptic patients had poor performance of a similar extent in different cognitive (as in scoring for absurdities, total verbal reasoning, memory for objects, bead memory and nonverbal short-term memory) and behavioral (as in scoring for depression, aggression and neurosis) functions testing. The effect of AEDs on cognition has been attributed to the fact that AEDs not only reduce neuronal irritability but also may impair neuronal excitability, neurotransmitter release, enzymes and factors critical for information processing and memory. Several previous studies indicated that AEDs have reversible and sometimes cumulative cognitive adverse consequences. ${ }^{33,34}$ Treated patients demonstrated worse scores in memories for digits forward, backward, and total verbal short-term memory) compared to untreated epileptic patients. The duration of AED intake was found to be associated with memory of objects, bead memory and total nonverbal short-term memory. In this study, no significant correlation was identified between cognitive functions and the dose of the AEDs. The number of utilized antiepileptic medications was significantly correlated with testing scores for absurdities.

In this study, no differential effect was identified between CBZ versus VPA therapy. CBZ utilization may be associated with mild cognitive dysfunction including excessive sedation, compromised attention, concentration and visual motor coordination, and psychomotor slowing. EEG slowing associated with CBZ might be significantly related to the magnitude of cognitive decline on later IQ subset performance. ${ }^{35}$ Studies revealed that children exposed to VPA in utero had learning difficulties, behavioral problems and increased need for special education. ${ }^{36}$ In one large prospective study, increased memory deficits, reduced verbal IQ by 8 to 15 points and an excess of additional needs were reported in children exposed to VPA prenatally. ${ }^{37}$ The cognitive side effects of CBZ have been attributed to their $\mathrm{Na}^{+}$channels blockade. ${ }^{38}$ VPA's modest effect on cognition has been attributed to the indirect modulation of GABA neurotransmissions. An enhancement of $\mathrm{GABA}_{\mathrm{A}}$ receptor-mediated hyperpolarizing responses caused by VPA will inhibit the activation of NMDA receptors in a dose-related manner and VPA reduces repetitive neuronal firing via blockade of voltage-dependant sodium $\left(\mathrm{Na}^{+}\right)$ channels. ${ }^{39,40}$ Its suppressive effect on synaptic response mediated by NMDA receptors may contribute to the impairment of long-term potentiation (LTP) and long-term depression (LTD) caused by VPA. VPA suppresses the expression of LTP in the CA1 region of the hippocampal slices. ${ }^{41} \mathrm{VPA}$ suppresses response mediated by NMDA receptors in a dose-related manner rat amygdaloid slices. ${ }^{42}$

The results of this study indicate that patients on conventional AEDs are at increased risk of behavioral abnormalities. In support, both treated and untreated epileptic patients had a similar extent of poor performance in behavioral functions testing (as in scoring for depression, aggression and neurosis). The dose of AEDs was significantly associated with testing scores for neurosis, verbal aggression and nonverbal 


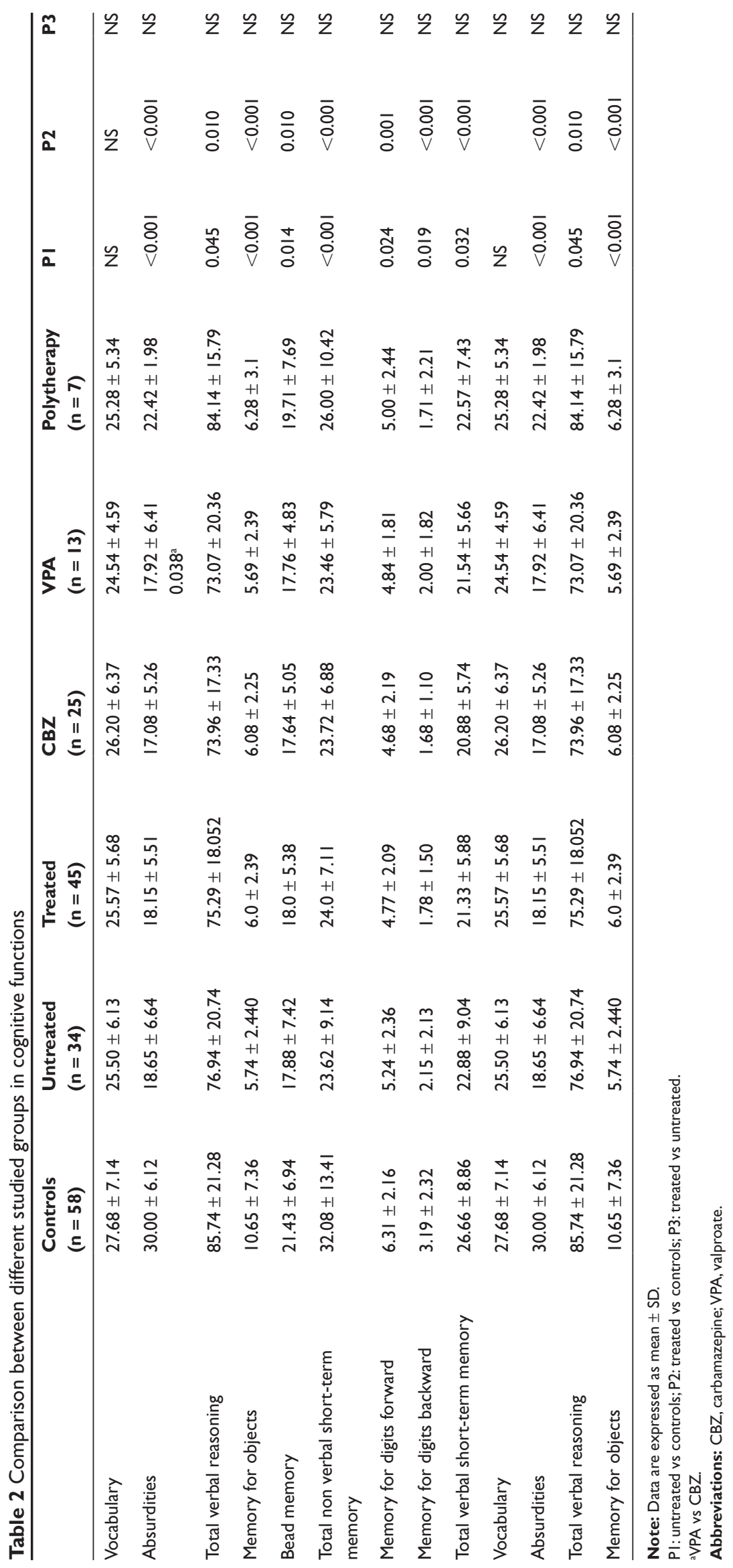


aggression, and the duration of AED intake was significantly associated with testing scores for depression, psychosis and nonverbal aggression. The relationship between AEDs and depression, aggression and neurosis in our group of patients could be attributed to psychosocial variables. The stigma resulting from epilepsy and learning problems may lower the parental and teacher expectations, scholastic achievement, educational levels rates of employment and hence poor quality of life, which are the long-term consequences in adults with epilepsy. ${ }^{43,44}$ But we cannot exclude the fact that depression, neurosis and psychosis in epilepsy might result from biological causes (epilepsy itself) in addition to reactive factors including AEDs. Functional neuroimaging studies (eg, PET and SPECT) have shown a relationship between heightened depression and hypometabolism in epileptic patients. ${ }^{45}$ The risk of cognitive side effects should be reduced by using monotherapy whenever possible, staying within standard therapeutic ranges for AED blood levels, and adhering to recommended drug dosages and titration rates. ${ }^{31}$ Neuropsychological side effects generally emerge according to a dose-dependent relationship. ${ }^{29}$ However, both quality of life ${ }^{35}$ and memory are affected, even when serum blood concentrations are within standard therapeutic ranges.

In summary, the results of this study provide a support for the notion that conventional AEDs are associated with poor performance in cognition, mood and behavior in patients with epilepsy. Duration of drug intake and the number of utilized AEDs are the main confounding variables. This study did not provide clues on how to exclude the effect of epilepsy itself and psychosocial variables as additional important confounding variables.

\section{Disclosures}

The authors declare no conflicts of interest.

\section{References}

1. Hauser, W.A. Incidence and prevalence. In: Engel J, Pedley TA, eds. Epilepsy: A Comprehensive Textbook. Philadelphia: Lippincott-Raven; 1997:47-57.

2. Gokcay A, Celebisoy N, Gokcay F, Atac C. Cognitive functions evaluated by $\mathrm{P} 300$ and visual and auditory number assays in children with childhood epilepsy with occipital paroxysms (CEOP). Seizure. 2006; 15:22-27.

3. Kälviäinen R, Äikiä M, Helkala EL, Mervaala E, Riekkinen PJ. Memory and attention in newly diagnosed epileptic seizure disorder. Seizure. 1992;1:255-262.

4. Kotloski R, Lynch M, Lauersdorf S, Sutula T. Repeated brief seizures induce progressive hippocampal neuron loss and memory deficits. Prog Brain Res. 2002;135:95-110.

5. Meador KJ. Cognitive effects of epilepsy and of antiepileptic medications. In: Wyllie E, ed. The Treatment of Epilepsy: Principles and Practices. Philadelphia: Lippincott Williams \& Wilkins; 2005:1185-1195. 
6. Piazzini A, Canevini MP, Turner K, Chifari R, Canger R. Elderly people and epilepsy: cognitive function. Epilepsia. 2006;47 Suppl 5:82-84.

7. Hamed SA. The aspects and mechanisms of cognitive alterations in epilepsy: the role of antiepileptic medications. CNS Neurosci Ther. 2009;15:134-156.

8. Aldenkamp AP, Overweg J, Gutter T, Beun AM, Diepman L, Mulder OG. Effect of epilepsy, seizures and epileptiform EEG discharges on cognitive function. Acta Neurol Scand. 1996;93:253-259.

9. Helmstaedter C, Kurthen M, Lux S, Reuber M, Elger CE. Chronic epilepsy and cognition: a longitudinal study in temporal lobe epilepsy. Ann Neurol. 2003;54:425-432.

10. Oyegbile TO, Dow C, Jones J, et al. The nature and course of neuropsychological morbidity in chronic temporal lobe epilepsy. Neurology. 2004;62:1736-1742.

11. Fisher JE, Vorhees C. Developmental toxicity of antiepileptic drugs: relationship to postnatal dysfunction. Pharmacol Res. 1992; 26:207-221

12. Yu XM, Salter MW. Gain control of NMDA-receptor currents by intracellular sodium. Nature. 1998;396:469-474.

13. Bradford HF. Glutamate, GABA and epilepsy. Prog Neurobiol. 1995;47:477-511.

14. International League Against Epilepsy. Proposal for revised clinical and electroencephalographic classification of epileptic seizures. Epilepsia 1981;22:489-501.

15. Goma A, Abdel-Regal S, Abdellah M, Hamed S. Monitoring antiepiletptic drug therapy in Upper Egypt: A retrospective 6-years experience in carbamazepine. J Egypt Soc Pharmacol Exp Ther. 2004; 25:319-335.

16. Wechsler D. WAIS-R Manual, Wechsler Adults Intelligence ScaleRevised. New York: Harcourt; 1981.

17. Fahmy SI, El-Sherbini AF. Determining simple parameters for social classification for health research. The Bulletin of the High Institute of Public Health. 1983:V111(5).

18. Melika LK. The Stanford. Binet Intelligence Scale, fourth edition. Arabic Examiner's Handbook. Cairo: Dar El Maref Publishing; 1983.

19. Delany EA, Hopkins TF. The Stanford. Binet Intelligence Scale, fourth edition: Examiner's Handbook. Chicago. The Riverside Publishing Co; 1986.

20. Steer R, Kumar G, Ranier W, Beck A. Use of the Beck Depression Inventory-II with Adolescent Psychiatric outpatients. J Psychopathol Behav Assess. 1998;20:127-137.

21. Gharyb AG. Beck Depression Inventory-II (BDI-II), Arabic Examiner's Handbook. Cairo: Dar El Anglo Publishing; 2000.

22. Eron LD, Gruder CL. Some topics closely related to the study of abnormal behavior: Aggression and fantasy. In: Reiss S, editor. Abnormality: Experimental and Clinical Approaches. New York: Macmillan. Publishing Co, 1977:579-638.

23. Elmestakway TA. Aggressive Behavior Scale. Cairo: Egyptian Books Publishing House; 2003

24. Eysenck HJ, Eysenck SB. Eysenck Personality Questionnaire (Junior and Adult), Great Britain. Sevenoaks: Hodder and Stoughton Educational; 1978.

25. Abd_Elkhalek A. Arabic form of Eysenck Personality Questionnaire (Junior and Adult), Alexandria: Dar El-Marifa Al-Jamiiyah; 1997.
26. Coenen AML, Konings GLMG, Aldenkamp AP, Renier WO, van Luijtelaar ELJM. Effects of chronic use of carbamazepine and valproate on cognitive processes. Epilepsy. 1995;8:250-254.

27. Shehata GA, Bateh AM. Cognitive function, mood, behavioral aspects, and personality traits of adult males with idiopathic epilepsy. Epilepsy Behav. 2009;14:121-124.

28. Bornstein RA, Pakalnis A, Drake ME Jr, Suga LJ. Effects of seizure type and waveform abnormality on memory and attention. Arch Neurol. 1988;45:884-887.

29. Binnie CD. Cognitive Impairment during epileptiform discharges: is it ever justifiable to treat the EEG? Lancet Neurol. 2003;2:725-730.

30. Butler CR, Zeman AZ. Recent insights into the impairment of memory in epilepsy: transient epileptic amnesia, accelerated longterm forgetting and remote memory impairment. Brain. 2008;131(9): 2243-2263.

31. Thom M. Neuropathological findings in epilepsy. Current Diagnostic Pathology. 2004;10:93-105.

32. Hamed SA. Neuronal plasticity: implications in epilepsy progression and management. Drug Dev Res. 2007;68:498-511.

33. Jokeit H, Kramer G, Ebner. Do antiepileptic drugs accelerate forgetting? Epilepsy Behav. 2005;6:430-432.

34. Drane DL, Meador KJ. Cognitive toxicity of antiepileptic drugs. In: Devinsky O, Westbrook LE, eds. Epilepsy and Developmental Disorders. Boston: Butterworth-Heinemann; 2002:311-330.

33. Mecarelli O, Vicenzini E, Pulitano P, et al. Clinical, cognitive and neurophysiologic correlates of short-term treatment with carbamazepine, oxcarbazepine and levetiracetam in healthy volunteer. Ann Pharmacother. 2004;38:1816-1822.

36. Moore SJ, Turnpenny P, Qinn A. A Clinical study of 57 children with fetal anticonvulsant syndromes. J Med Genet. 2000;37:489-497.

37. Adab N, Kini U, Vinten J, et al. The longer term outcome of children born to mothers with epilepsy. J Neurol Neurosurg Psychiatry. 2004;75:1575-1583.

38. Temkin NR. Antiepileptogenesis and seizure prevention trials with antiepileptic drugs: meta-analysis of controlled trials. Epilepsia. 2001;42:515-524.

39. Löster W. Basic pharmacology of valproate. CNS Drugs. 2002;16:669694.

40. vanDongen AM, VanErp MG, Voskuyl RA. Valproate reduces excitability by blockage of sodium and potassium conductance. Epilepsia. 1986;27:177-182.

41. Lee GY, Brown LM, Teyler TJ. The effects of anticonvulsant drugs on long-term potentiation (LTP) in the rat hippocampus. Brain Res Bull. 1996;39:39-42.

42. Gean PW, Huang CC, Huang CR, Tsai JJ. Valproic acid suppresses the synaptic response mediated by the NMDA receptors in rat amygdalar slices. Brain Res Bull. 1994;33:333-336.

43. Kanner AM, Nieto JCR. Depression disorder in epilepsy. Neurology. 1999;53 Suppl 2:S26-S32.

44. Tracy JI, Lippincott C, Mahmood T, et al. Are depression and cognitive performance related in temporal lobe epilepsy? Epilepsia. 2007;48:2327-2335

45. Loring DW, Marino S, Meador KJ. Neuropsychological and behavioral effects of antiepilepsy drugs. Neuropsychol Rev. 2007;17:413-425.

Neuropsychiatric Disease and Treatment

\section{Publish your work in this journal}

Neuropsychiatric Disease and Treatment is an international, peerreviewed journal of clinical therapeutics and pharmacology focusing on concise rapid reporting of clinical or pre-clinical studies on a range of neuropsychiatric and neurological disorders. This journal is indexed on PubMed Central, the 'PsycINFO' database and CAS, and is the official 